\title{
Supporting Information: Boron-Loaded Polymeric Sensor for the Direct Detection of Thermal Neutrons
}

Prodromos Chatzispyroglou, Joseph L. Keddie, and Paul J. Sellin*

Department of Physics, University of Surrey, Guildford, UK

Email: p.j.sellin@surrey.ac.uk

\section{Neutron Sensitivity}

The quantum efficiency (QE) of the sensor is determined by the interaction probability of the thermal neutrons with the boron particles:

$$
Q E=P(n, t)=1-e^{-\sigma \cdot n \cdot t}
$$

where $\sigma$ is the thermal neutron microscopic cross section $\left(3,840\right.$ barns for $\left.{ }^{10} \mathrm{~B}\right), n$ is the atomic density of boron within the nanocomposite volume (not the atomic density within an individual B nanoparticle) and $t$ is the thickness of the active region. Using Eq.1, a $10 \mu \mathrm{m}$ thick PTAA film filled with ${ }^{10} \mathrm{~B}$ at a density of $10^{22}$ atoms $\mathrm{cm}^{-3}$ will have a QE of $4 \%$. Increasing the thickness of the film to $100 \mu \mathrm{m}$ 
at the same boron density (requiring additional boron to be added) will increase the QE to $32 \%$. In this work, we used naturally-occurring boron in the nanoparticles, because isotopically-enriched ${ }^{10} \mathrm{~B}$ nanoparticles are not readily available. In this case, the effective neutron cross-section is $20 \%$ of the ${ }^{10} \mathrm{~B}$ value.

The magnitude of the response current $I_{\text {resp }}$ is given by:

$$
I_{\text {resp }}=\Phi_{n_{t h}} \cdot A_{\text {dev }} \cdot P_{\text {int }} \cdot Q_{i o n} \cdot \epsilon
$$

where $\Phi_{n_{t h}}$ is the flux of thermal neutrons, $A_{d e v}$ is the active area of the device (equal to the area of the top electrode), $P_{\text {int }}$ is the interaction probability of a thermal neutron in the sensor (from Eq. 1), $Q_{i o n}$ is the charge created in the film from the daughter ions, and $\epsilon$ is the charge collection efficiency.

The created charge $Q_{i o n}$ is proportional to the energy deposited in the film by the daughter ions $E_{\text {dep }}$ such that:

$$
Q_{i o n}=\frac{E_{d e p} q}{W}
$$

where $W$ is the 'electron hole pair creation energy' which is a characteristic value for different sensor materials, and $q$ is the electronic charge. To estimate the typical thermal neutron response current, we assume an $E_{d e p}$ value which is the mean of the two daughter ion energies $(1.16 \mathrm{MeV})$. The band gap of undoped PTAA films has been previously reported as $2.95 \mathrm{eV}$, and we estimate the $W$ parameter for PTAA using the approximation $W=2.8 \times E_{g}$, giving $8.3 \mathrm{eV}$ per electron-hole pair. Therefore the estimated charge created per neutron interaction $Q_{\text {ion }}$ is $\sim 2.2 \times 10^{-14} \mathrm{C}$.

Assuming estimated values of $10 \%$ for the charge collection efficiency and of $4 \%$ for the QE, Equation 3 gives an estimated value of $100 \mathrm{pA}$ for the neutron-induced photocurrent from our device with a neutron flux of $10^{6}$ neutrons $\mathrm{cm}^{-2} \mathrm{~s}^{-1}$. 


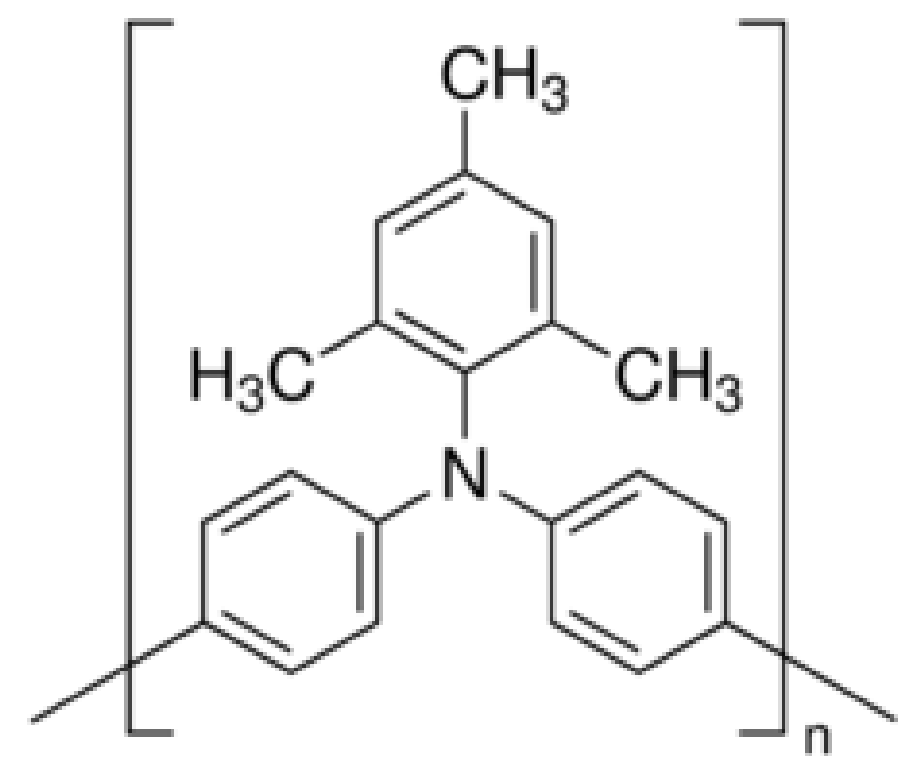

Figure S1: Chemical structure of the repeat unit of poly(bis(4-phenyl) (2,4,6-trimethyl-phenyl) amine) or PTAA

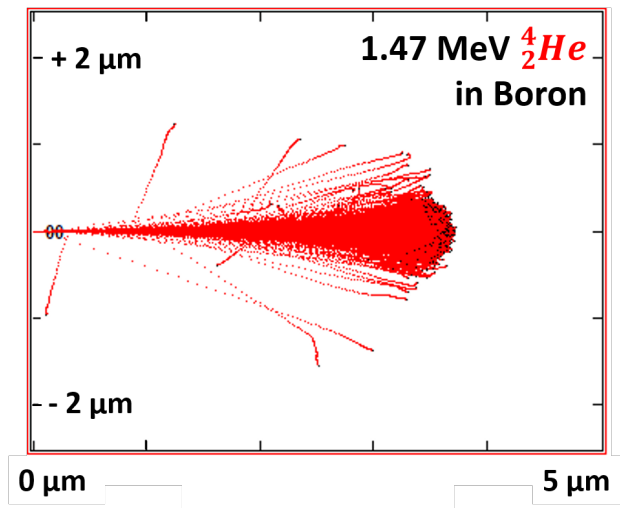

(a)

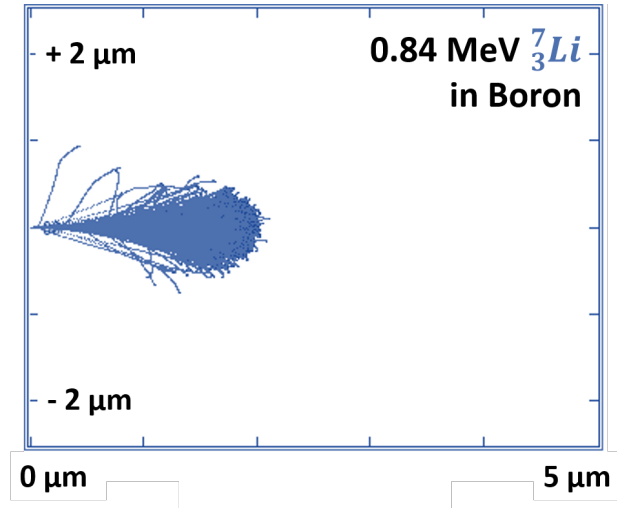

(b)

Figure S2: Monte Carlo modelling of the projected range of (a) ${ }_{2}^{4} \mathrm{He}$ and (b) ${ }_{3}^{7} \mathrm{Li}$ daughter ions in boron, calculated using the SRIM code. A thermal neutron reaction with ${ }^{10} \mathrm{~B}$ releases a pair of ${ }_{2}^{4} \mathrm{He}$ and ${ }_{3}^{7} \mathrm{Li}$ daughter ions with the indicated kinetic energies, which together sum to the reaction Q-value of $2.31 \mathrm{MeV}$. 


\section{Optical Microscopy}

Optical microscopy (Olympus) was used to assess the surface quality of the deposited films. In the earlier work using polymers with relatively low molecular weight, the films were brittle with cracks forming during the deposition and drying process (Figure S3).

During the optimization of the boron-loaded films, and using the as-received boron nanoparticle dispersion, micrometer-sized boron agglomerates could be observed on the film surface, even at low boron loading $(0.85 \mathrm{vol} \%)$. The particles were large enough to be observed near the nanocomposite surface by reflectance optical microscopy, but it was not possible to obtain sub-surface structural information because of opacity.

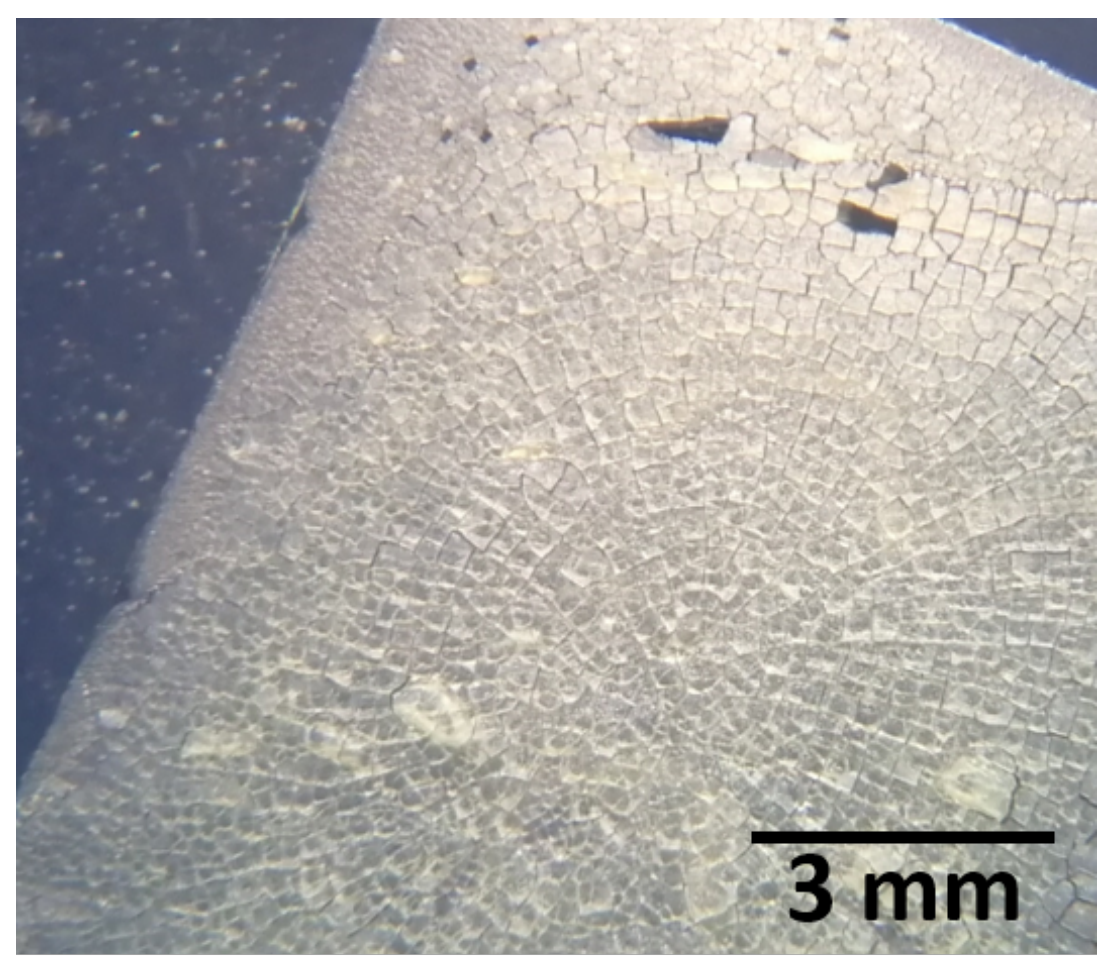

Figure S3: Digital photograph of crack formation in a brittle film deposited from a polymer with a low $M_{w}\left(16 \mathrm{~kg} \mathrm{~mol}^{-1}\right)$ 


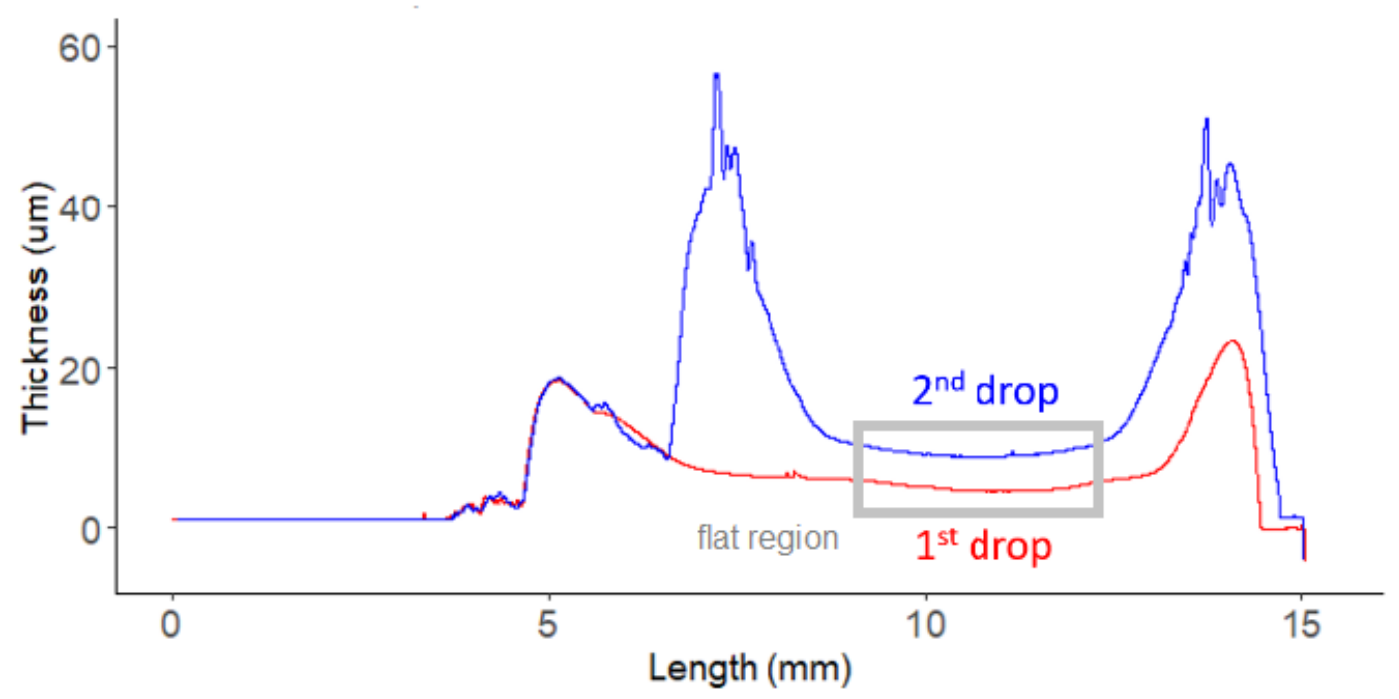

Figure S4: Typical height profile of PTAA films, demonstrating multilayer film deposition. The solid lines show the layers deposited after the first (red) and the second (blue) deposition of a $5 \mathrm{wt} \%$ PTAA $\left(M_{w}=350 \mathrm{~kg} \mathrm{~mol}^{-1}\right)$ solution. Notice that thicker films can be fabricated by stacking layers. There is pronounced 'knee' formation at the edges, and the measured thickness is averaged over the flat region in the centre of the film.

\section{IV and Photocurrent Data}

Figure S5 shows the optical photocurrent obtained from LED illumination of a neat PTAA sensor. The strong sensitivity to optical light, combined with the low dark current associated with the good rectification of the junction, gives a high signal/noise ratio and confirms the good charge transport properties of the PTAA layer. Figure S6(a) shows the electrical characteristics of working PTAA diodes containing varying boron loading (from 0 vol\% to 2 vol\%). As boron-loading increases, the J-E characteristics become more ohmic. Figure S6(b) shows the electrical characteristics of films deposited by the optimized process. It can be seen that, although the diminishing rectification issue was not resolved, higher loadings of B were achieved with the same leakage current. 


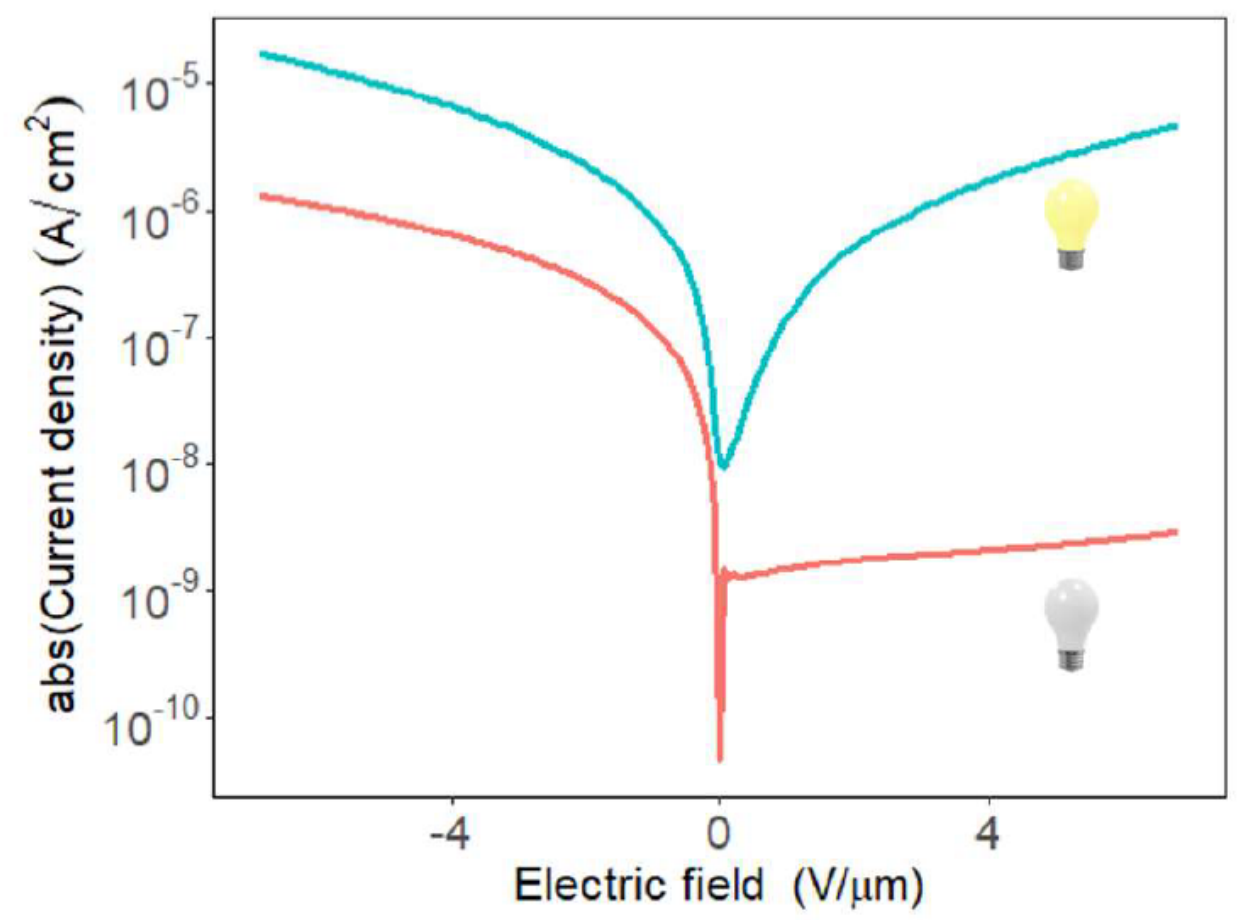

Figure S5: Optical photocurrent obtained from LED illumination of a PTAA sensor (blue line) and the dark current without illumination (red line) under forward and reverse bias. The PTAA's $M_{w}=$ $350 \mathrm{~kg} \mathrm{~mol}{ }^{-1}$ and its thickness is $14.6 \mu \mathrm{m}$. 


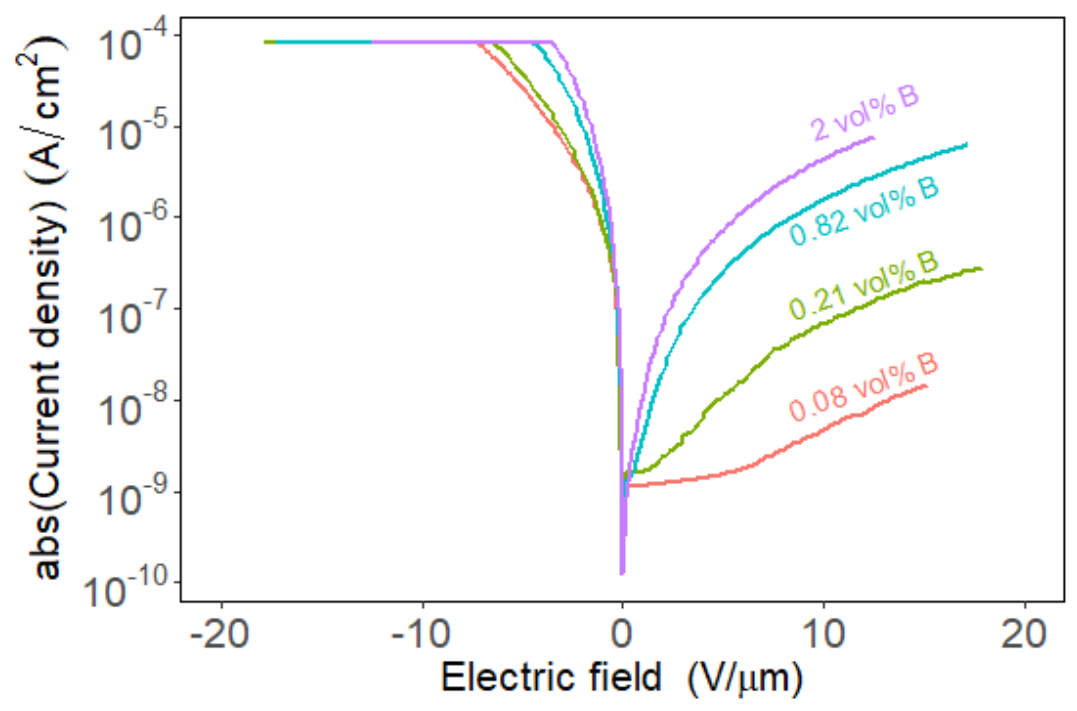

(a)

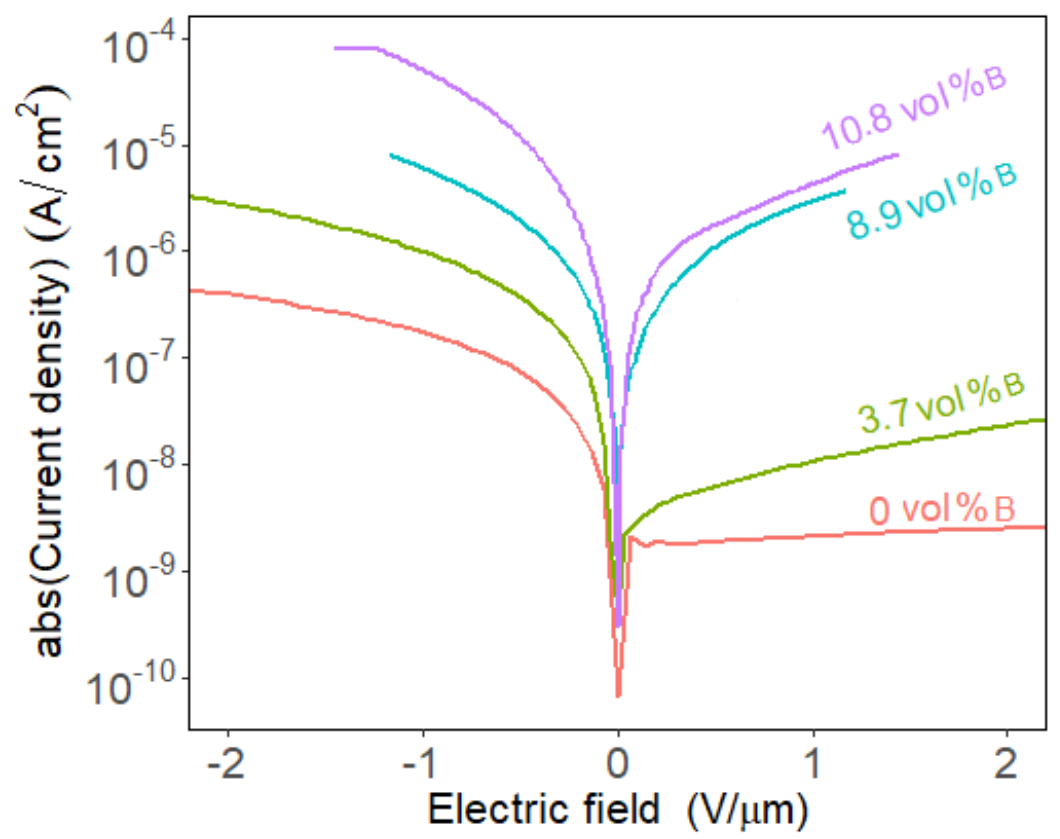

(b)

Figure S6: Current density as a function of the electric field for B-loaded PTAA nanocomposite films with increasing B content (as labelled) (a) without and (b) with the addition of Span80 surfactant and sonication of the B nanoparticles. In (a) $M_{w}=16 \mathrm{~kg} \mathrm{~mol}^{-1}$, and in (b) $M_{w}=350 \mathrm{~kg} \mathrm{~mol}^{-1}$. The same leakage current as in (a) has been found with a higher B loading. 


\section{Scanning Electron Microscopy}

Figure S7 shows typical SEM cross-sectional images of PTAA films deposited without the use of surfactants to disperse the boron nanoparticles (9.6 vol \%). When not dispersed by the surfactants in the solvent, the boron nanoparticles have a tendency to sediment during film formation. Consequently, a layer of sedimented B nanoparticles can be observed along the interface with the substrate.

Figure S8 compares SEM images of four nanocomposite films, each one having a different treatment of the original boron dispersion prior to the addition of the polymer. Detailed information about the films can be found in Table S1. The quoted thickness was measured at the region where the contacts are fabricated. SEM images show embedded boron particles in the polymeric film. These particles look like shapeless details in a solid background color and are distinguishable due to their contrast. Later elemental analysis based on WDS associated these structures with elemental boron.

Film 1 (Figure S8a) shows boron agglomerates of about $20 \mu \mathrm{m}$ in diameter on the surface of the film. In this film, the presence of agglomerates enhances the development of cracks. Agglomerates of this size are typical for films that used the original B dispersion as received. Figure S8b and S8c show two films that were fabricated side by side having different Span80:B mass ratios (1:10, in Figure S8b against 2:5, in Figure S8c). After the addition of Span80, both dispersions were bath-sonicated for $10 \mathrm{~min}$, before adding PTAA $(\mathrm{Mw}=16 \mathrm{~kg} / \mathrm{mol})$. Although, the lower Span80 concentration reduced the size of agglomerates, clusters in the order of $10 \mu \mathrm{m}$ in size (Figure S8b inset) were identified. Increasing the Span80:B mass ratio to 2:5 reduced the size of agglomerates by one order of magnitude (Figure S8c). Spectral analysis, conducted in the region designated by the red square, shows an increased count rate for the $K_{\alpha}$ characteristic line of boron and a partial separation from the $K_{\alpha}$ characteristic line of carbon (Figure S8d inset). Carbon also has a high count rate due to the high carbon atom density in the polymer. False positive recordings are depicted in the elemental map of boron (Figure S8d) due to partial overlap of the B-C $K_{\alpha}$ peaks. Nevertheless, 
the boron concentration map matches the position of the structures shown in the SEM image, which results in an undoubted identification of boron particles. Despite the small size of agglomerates, the dispersion is not very homogeneous.

A further reduction of the size of agglomerates was achieved by increasing the Span80:B ratio, as was shown by dynamic light scattering in dilute suspensions of boron in toluene. Figure S8(e) shows the result of the fully optimized fabrication process. The large increase in the film thickness, the amount of $\mathrm{B}$, and the homogeneity of its dispersion is depicted in the comparison between Figure S8(e) against Figure S8(c) and $\mathbf{S 8 ( d )}$. Using the high- $M_{w}$ PTAA allowed for the deposition of thicker films (here $45.4 \mu \mathrm{m}$ ). The use of 2:5 Span80:B mass ratio followed by 30-minute bath sonication led to a nanocomposite with a very homogeneous distribution of boron nanoparticles.

Table S1: Details of films shown in Figure S8.

\begin{tabular}{||c|c|c|c|c|c||}
\hline & $\begin{array}{c}\text { Thickness } \\
(\mu \mathrm{m})\end{array}$ & Polymer $M_{w}(\mathrm{~kg} / \mathrm{mol})$ & $\begin{array}{c}\text { B-loading } \\
(\% \text { wt. })\end{array}$ & $\begin{array}{c}\text { Span80:B } \\
\text { mass } \\
\text { ratio }\end{array}$ & $\begin{array}{c}\text { Sonication } \\
\text { time } \\
(\mathrm{min})\end{array}$ \\
\hline Film 1 & - & 16 & 1.99 & $0: 1$ & 0 \\
\hline Film 2 & 2.4 & 16 & 2.36 & $1: 10$ & 10 \\
\hline Film 3 & 3.5 & 16 & 2.35 & $2: 5$ & 10 \\
\hline Film 4 & 45.4 & 350 & 19.10 & $2: 5$ & 30 \\
\hline
\end{tabular}




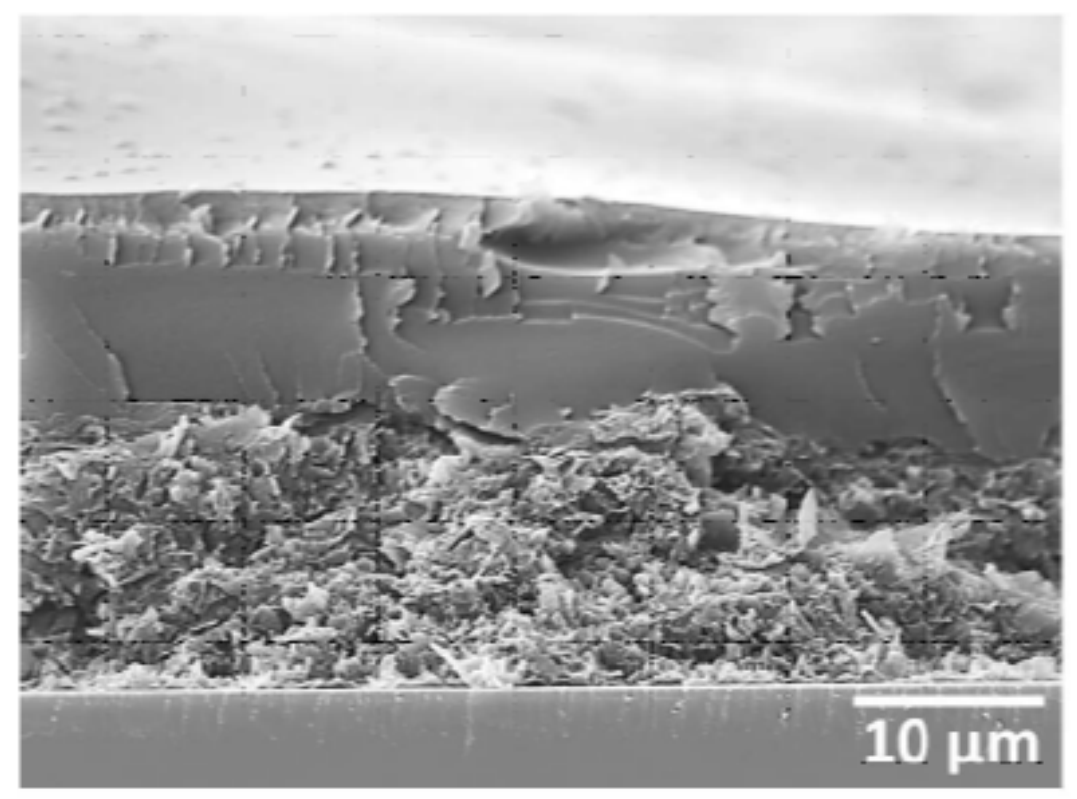

Figure S7: SEM image of cross-section of film fabricated without the use of surfactants to disperse the B nanoparticles (present at $9.6 \mathrm{vol} \%$ ). There is a sedimented layer of B nanoparticles along the interface with the substrate. (Image processed with $+40 \%$ Contrast, $+50 \%$ Sharpness) 


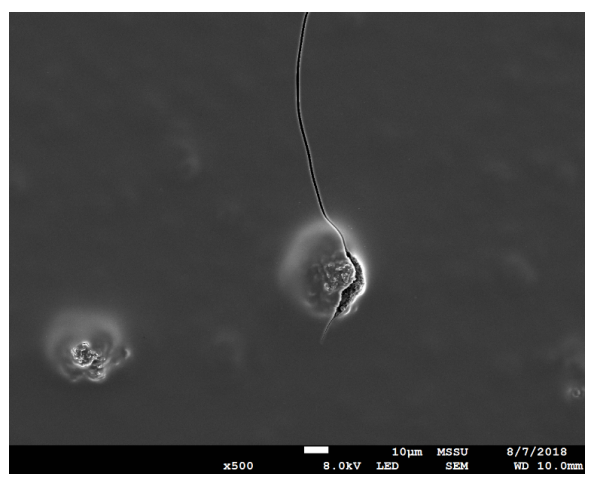

(a)

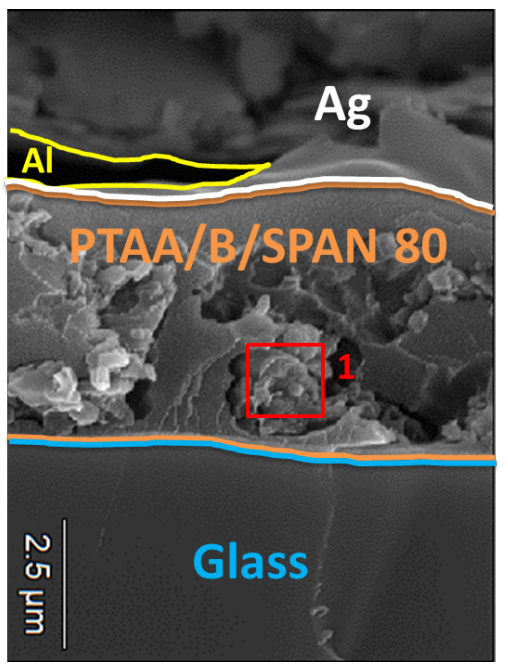

(c)

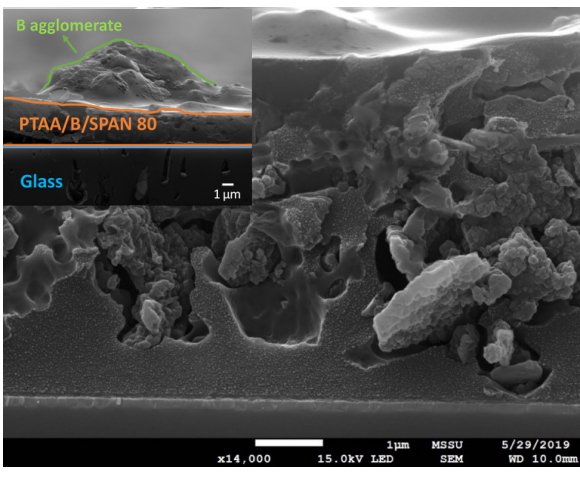

(b)

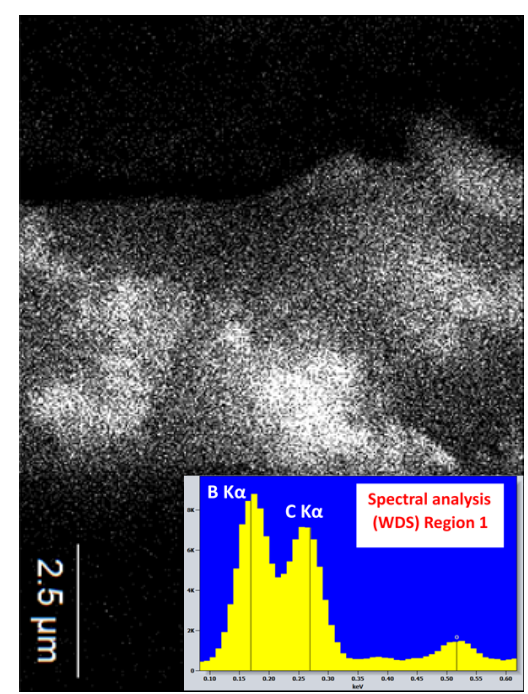

(d)

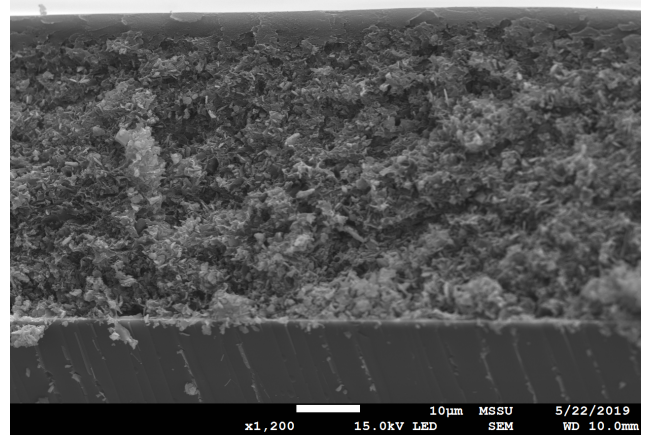

(e)

Figure S8: SEM images of cross-sections of the PTAA films described in Table S1 with Span80:B mass ratios of (a) 0:1 (Film 1); (b) 1:10 (Film 2); (c) 2:5 (Film 3); (d) Boron map of Film 3, obtained from WDS analysis; (e) 2:5 (Film 4) with 19.1 wt.\% B and sonication for 30 min. 


\section{Neutron Detection}

The experimental configuration of the sensor during the neutron measurements is shown in Figure S9. A thin-walled aluminum enclosure was used to minimize neutron scattering or additional reactions in the beam. The polymer sensor was mounted onto a removable socket on a printed circuit board. Low noise current data of three boron-loaded sensors (see Table 1 in the main paper) were recorded before and during thermal neutron exposures. Low leakage current yields higher neutron-signal-todark-current ratios, which is an index of neutron signal resolution, and this is typically achieved at low operating bias where the dark current is minimized. Neutron signal could be resolved from both the electronic noise and the dark current. The magnitude of the noise was at the pA level, that is one order of magnitude less than the neutron signal. Hence, it did not affect the measurement. The neutron signal could be clearly resolved from the dark current, despite being two orders of magnitude lower in amplitude. For example, at the maximum neutron beam output, the response current was approximately $0.05 \mathrm{nA}$, at $10 \mathrm{~V}$ bias.

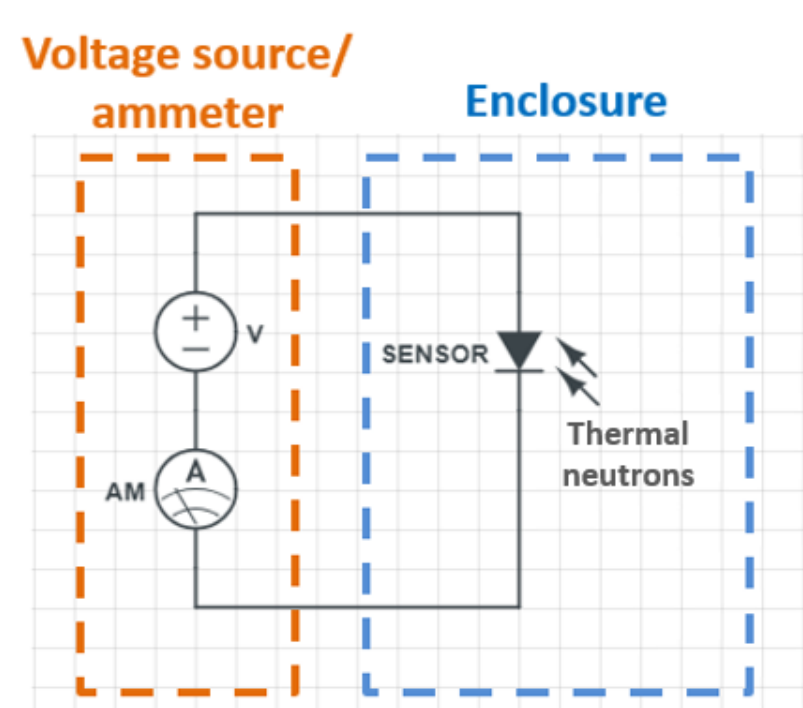

(a)

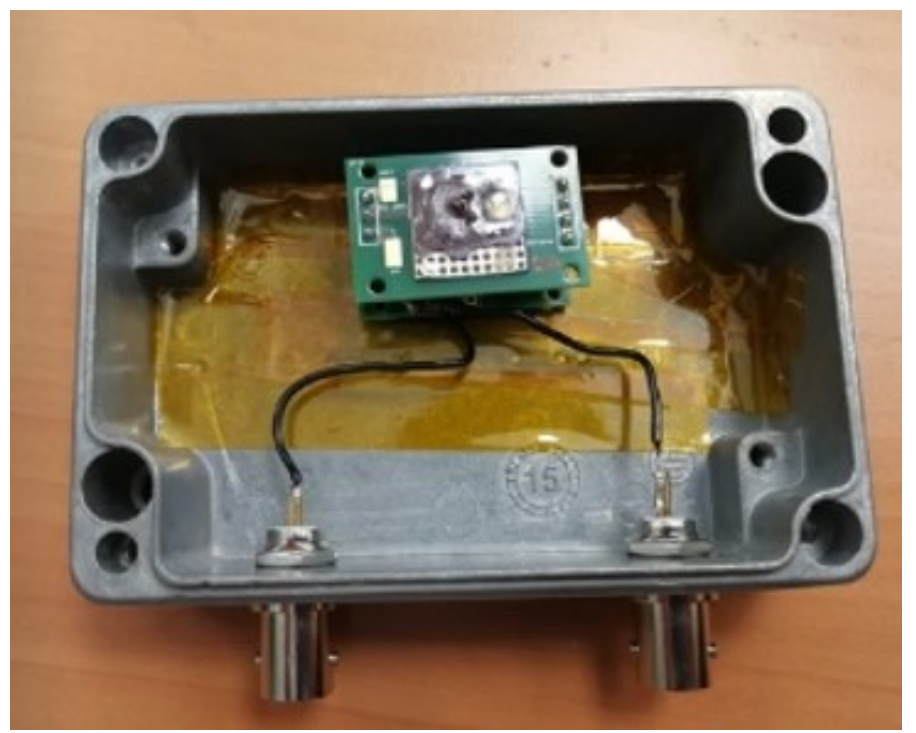

(b)

Figure S9: (a) Experimental configuration during neutron exposures. (b) Aluminum enclosure showing the polymer sensor mounted on a printed circuit board. 